\title{
Synthesis and Characterization of Photoresponsive Biopolymers Having Spiropyran and Chitosan Groups
}

\author{
Yuan-Jing $Z \mathrm{HOU}^{1, a}$, Qing $X U^{2, b,{ }^{*}}$, Qiang $\mathrm{JIA}^{2}$, Fei $\mathrm{NIE}^{2}$, Gui-Long $\mathrm{GAO}^{2}$ and Ying \\ ZHANG $^{1}$ \\ ${ }^{1}$ Guizhou Academy of Analysis and Testing, Guiyang 550002, Guizhou, P. R. China \\ ${ }^{2}$ Institute of Biology, Guizhou Academy of Sciences, Guiyang 550009, Guizhou, P. R. China \\ aemail: zhouyuanjing-8006@163.com, bemail: xqhaoy@163.com \\ *Corresponding author: Qing Xu
}

Keywords: Photoresponsive, Spiropyran, Chitosan, Synthesis, Characterization.

\begin{abstract}
Photoresponsive materials have attracted much attention recently because of their potential utility in many areas such as industrial, agricultural and pharmaceutical. In this paper, several photoresponsive biopolymers having spiropyran and chitosan groups were synthesized, and the structure and properties of the products were characterized by means of FTIR, H NMR, MS, and SEM. The photoresponsive properties were investigated by UV-Vis spectroscopy. They showed good photoresponsive properties, and may consider as photoresponsive materials for application in intelligent drug delivery system.
\end{abstract}

\section{Introduction}

Recently, photoresponsive polymers have attracted significant scientic technological interest because they could be used as optical switches, high-density optical data storage, displays, non-linear optics and other molecular devices [1]. Spiropyrans, as a class of typical photochromic compounds, respond to light and undergo a reversible isomerization between colorless spiropyran (SP) and colored merocyanine (MC) (Fig. 1). Numerous applications based on their reversible color and some changes in physical and chemical properties were suggested and examined [2-7]. Chitosan, as the second most abundant polysaccharide, next to cellulose, has considerable application in food processing, cosmetics, environmental protection, wastewater management, agriculture, pharmaceutical, and biomaterial [8-12].

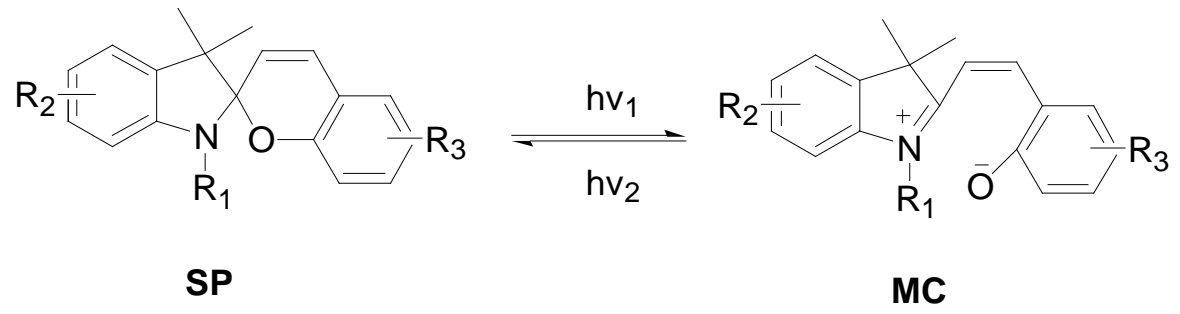

Fig. 1. Photochromism of spiropyran between the states: spiropyran (SP) and merocyanine (MC)

Herein a novel photoresponsive polymer having spiropyran and chitosan groups was constructed. The simple grafting reaction and the photoresponsive properties of products were showed. The results may contribute to promoting the application of spiropyran modified with chitosan as molecular switches in photoresponsive drug delivery system. 


\section{Experimental}

\section{Reagents and Method}

Chitosan obtained from Shanghai Bo'ao Biotechnology Co. (Shanghai, China) with viscosity-average molecular weight of $500 \mathrm{kDa}$ and $97 \%$ degree of deacetylation was purified before using. All other chemical reagents used in this experiment were obtained from commercial sources and were of the highest purity available. Fourier transform infrared spectroscopy (FTIR) tests were conducted on a Spectrum 100 FTIR spectrometer (PerkinElmer, US) using KBr discs. H NMR spectra were obtained on a Mercury 400 spectrometer $(400 \mathrm{MHz})$ using TMS as an internal standard. The scanning electron (SEM) microscopy images were recorded on a JMS-120 scanning electron microscopy. UV-Vis absorption spectra were recorded using a Lambda $35 \mathrm{UV}-\mathrm{Vis}$ spectrophotometer (PerkinElmer, US).

\section{Synthesis and Characterization}

Fig. 2 showed the synthetic route of compounds.

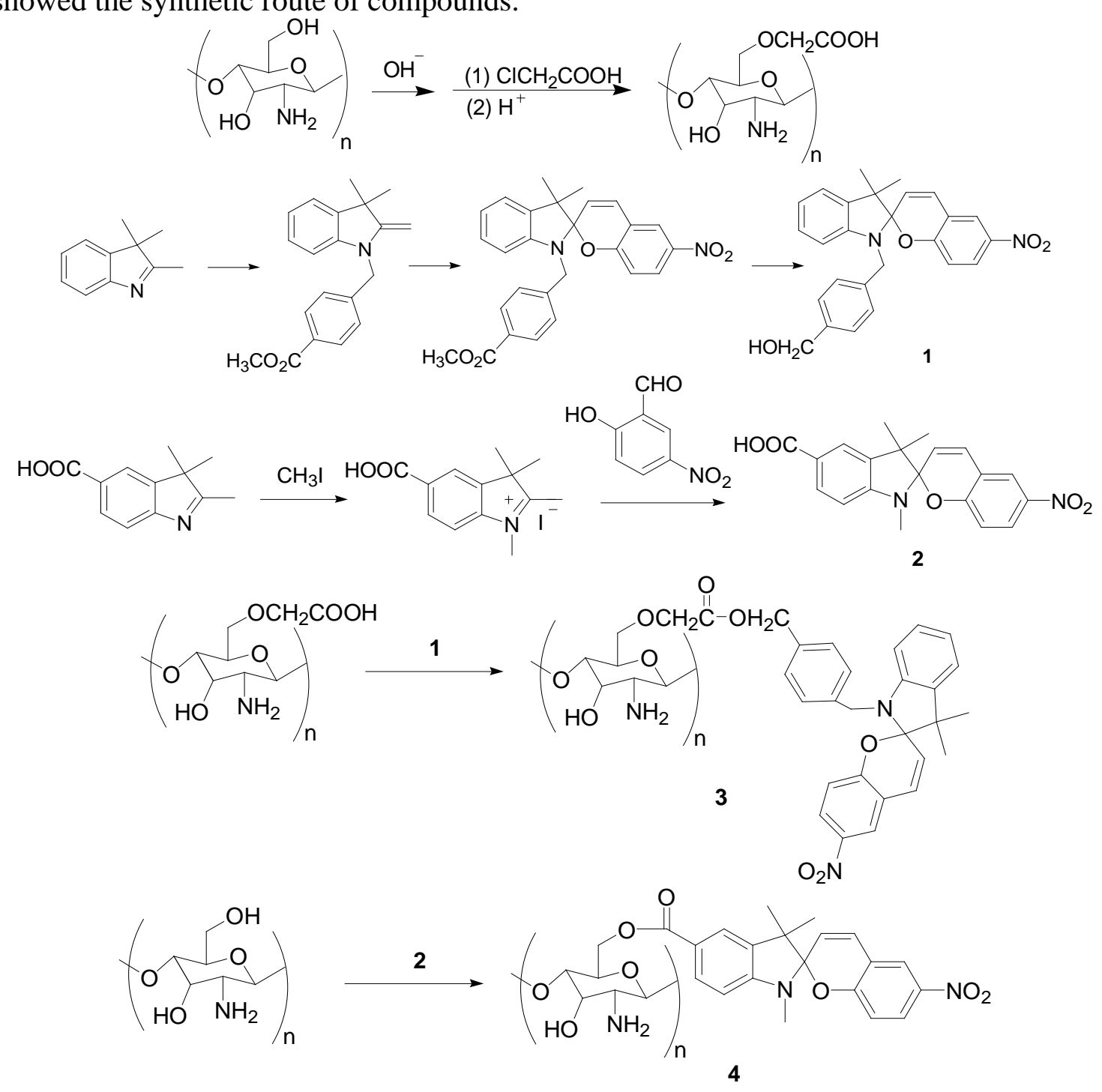

Fig. 2. Synthetic routes of compounds

\section{Modification of Chitosan}

Under alkaline conditions, chitosan and chloroacetic acid reacted at $10^{\circ} \mathrm{C}$ for $24 \mathrm{~h}$. After acid treatment, carboxymethyl chitosan was obtained [13]. The substitution degree of carboxymethyl was 
0.88. FTIR (KBr, $\left.\mathrm{cm}^{-1}\right)$ : 3421, $1720(\mathrm{COOH}), 1644(\mathrm{C}=\mathrm{O}), 1408(\mathrm{C}-\mathrm{O}), 1067\left(\mathrm{NH}_{2}\right), 1245(\mathrm{C}-\mathrm{N})$, 1028.

\section{Synthesis of Spiropyran Derivative Compounds}

1’-(4-Hydroxymethylbenzyl)-6-nitroindolinospirobenzopyran (1)

According to the literature [14], compound 1 was synthesized with $80 \%$ yield. ${ }^{1} \mathrm{H}$ NMR $\left(\mathrm{CDCl}_{3}\right.$, $400 \mathrm{MHz}) \delta: 8.05(1 \mathrm{H}, \mathrm{d}), 7.99(1 \mathrm{H}, \mathrm{d}), 7.31-7.26(4 \mathrm{H}, \mathrm{m}), 7.15(1 \mathrm{H}, \mathrm{d}), 7.09(1 \mathrm{H}, \mathrm{t}), 6.90(1 \mathrm{H} \mathrm{t}), 6.88$ $(1 \mathrm{H}, \mathrm{d}), 6.79(1 \mathrm{H}, \mathrm{d}), 6.36(1 \mathrm{H}, \mathrm{d}), 5.89(1 \mathrm{H}, \mathrm{d}), 4.69(2 \mathrm{H}, \mathrm{d}), 4.52(1 \mathrm{H}, \mathrm{d}), 4.23(1 \mathrm{H}, \mathrm{d}), 1.62(1 \mathrm{H}, \mathrm{m})$, 1.36 (3H, s), 1.32 (3H, s,). MS (MALDI-TOF) m/z $428\left(\mathrm{M}^{+}\right)$.

5'-carboxyl-1'-methyl-6-nitroindolinospirobenzopyran (2)

According to the literature [15], compound 2 was synthesized with $78 \%$ yield. FTIR $\left(\mathrm{KBr}, \mathrm{cm}^{-1}\right)$ : 3435, 1670, 1615, 1578, 1519, 1346, 1258, 1093, 1009, 958, 818, 739. ${ }^{1} \mathrm{H}$ NMR (DMSO, $\left.400 \mathrm{MHz}\right)$ $\delta: 12.51(\mathrm{~s}, 1 \mathrm{H}), 8.25-8.24(\mathrm{~d}, 1 \mathrm{H}), 8.02-8.01(\mathrm{~d}, 1 \mathrm{H}), 7.84-7.82(\mathrm{~d}, 1 \mathrm{H}), 7.69(\mathrm{~s}, 1 \mathrm{H}), 7.29-7.25(\mathrm{~d}, 1 \mathrm{H})$, 6.94-6.91(d,1H), 6.72-6.69 (d,1H), 6.04-6.01(d,1H), 2.77 (s, 3H), 1.26 (s,3H), 1.14 (s, 3H).

\section{Synthesis of Graft Products}

Grafting spiropyran (1) or (2) to carboxymethyl chitosan or chitosan was conducted as: 5 mmol spiropyran and $5 \mathrm{mmol}$ purified carboxymethyl chitosan or chitosan were dissolved in $100 \mathrm{~mL}$ dichloromethane and stirred at room temperature for 10 minutes. 5 mmol $\mathrm{N}$, $\mathrm{N}$-dicyclohexylcarbodiimide and moderate 4-dimethylaminopyridine were added to the reaction solution and stirred at room temperature overnight. The solution was filtered and the solvent was evaporated. The obtained solid was dissolved in acetone, and then petroleum ether was added to the solution for precipitating product. The precipitating product was treated twice like this. The final product was kept in a desiccator at room temperature overnight.

3: FTIR (KBr, cm ${ }^{-1}$ ): 3442, 2925, 1738, 1645, 1580, 1344, 1258, 1135, 958, 818.

4: FTIR (KBr, $\left.\mathrm{cm}^{-1}\right)$ : 3440, 1736, 1638, 1565, 1342, 1235, 956, 808, 746.

\section{Result and Discussion}

\section{Scanning Electron Microscopy Images}
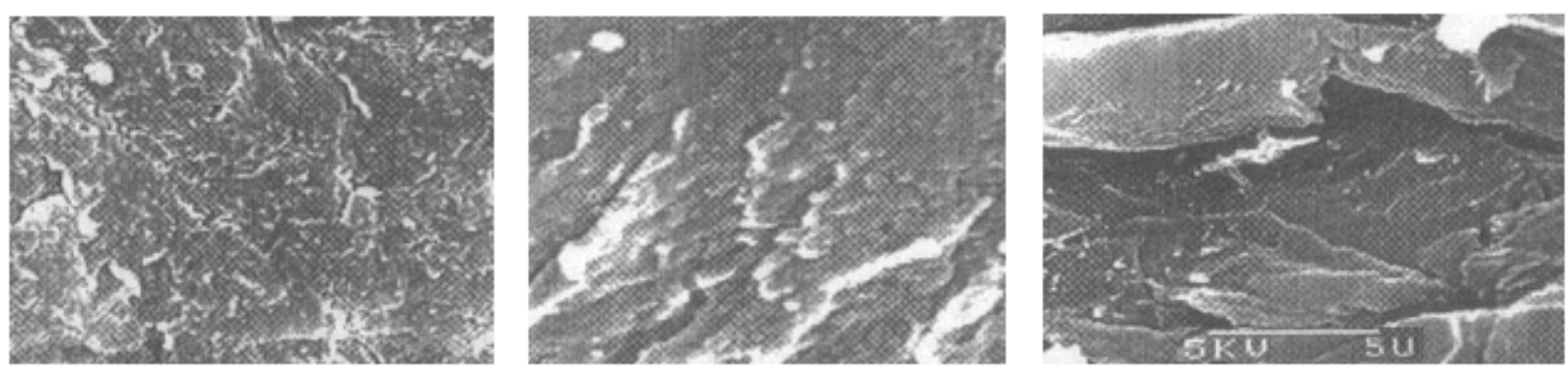

Fig. 3. The SEM images showing chitosan (left), carboxymethyl chitosan (middle) and spiropyran-graft-chitosan (right).

Fig. 3 showed the SEM images. Chitosan (left) had a higher degree of crystallization due to the strong intramolecular and intermolecular hydrogen bonds. The surface of the chitosan particle was compact and orderly. Carboxymethyl chitosan (middle) showed some small pieces due to the weakening hydrogen bonds. And the surface of the carboxymethyl chitosan particle was dense and smooth.The particle of esterified graft product (right) was different from the chitosan and carboxymethyl chitosan. It exhibited some cracks, which may be due to the introduction of spiropyran monomer on the side chain of carboxymethyl chitosan, destroyed the original crystal structure, and weakened the interaction of hydrogen bond. 


\section{Photochromic Properties}

The photochromic properties of compounds 1-4 were investigated. In organic solvent, they all showed good photochromic properties. Before light irradiation, they had absorption in the ultraviolet region (200-400 nm). After light irradiation, they appeared absorption in the visible region (400-800 $\mathrm{nm}$ ), and the absorption in the ultraviolet region weakened. Norri et al. considered that the absorption of near 210-250 nm was indoline portion, and near 310-350 nm was benzopyran ring. After UV irradiation, the $\mathrm{C}-\mathrm{O}$ bond of spiropyran ring was broke and generated merocyanine with a dipolar ionic structure, which could further generate quinone structure. The stability of the merocyanine affected by solvent polarity [16]. As a typical example, Table 1 gave the absorption maximum of compound 2 in different solvents after light irradiation.

Table 1 . The absorption maxima ( $\lambda \max )$ of the compound 2 in different solvents after irradiation for 30 minutes

\begin{tabular}{cccccc}
\hline Solvent & Ether & Tetrahydrofuran & Methylene chloride & Acetone & Ethanol \\
\hline$\lambda \max (\mathrm{nm})$ & 610 & 605 & 599 & 565 & 550 \\
\hline
\end{tabular}

Photochemical isomerization of compound 3 was demonstrated by UV-Vis absorption spectroscopy. An ethanol solution was prepared: $1.0-\mathrm{mg} 3$ was dissolved in $10 \mathrm{~mL}$ of ethanol, and the solution was filtered through a $0.22-\mathrm{mm}$ filter before use. Then the solution was irradiated with either UV light (365 nm, Fig.4 left) or visible light (620 nm, Fig.4 right) until photostationary states were reached. On UV irradiation, a strong absorption band at $545 \mathrm{~nm}$ appeared, which is characteristic of the MC form (Open-SP). Irradiation with visible light isomerized the MC form back to the SP form, as was confirmed by the spectra gradually reverting to the original absorption profile.

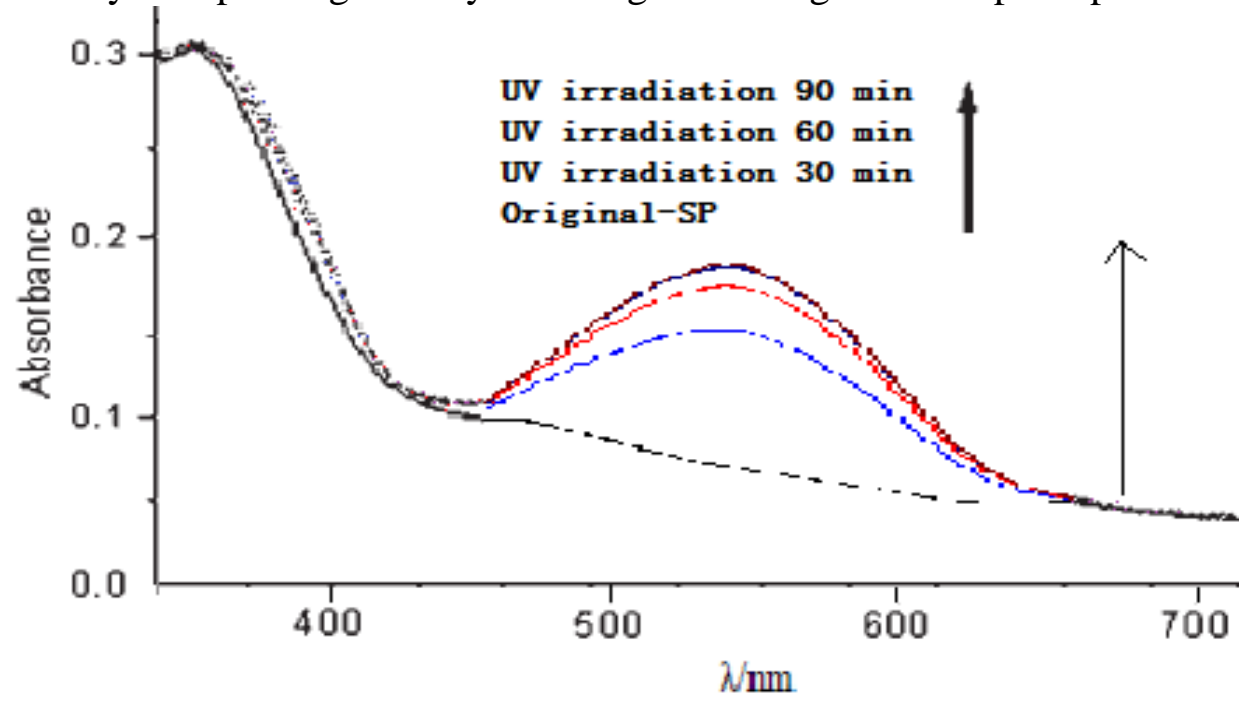




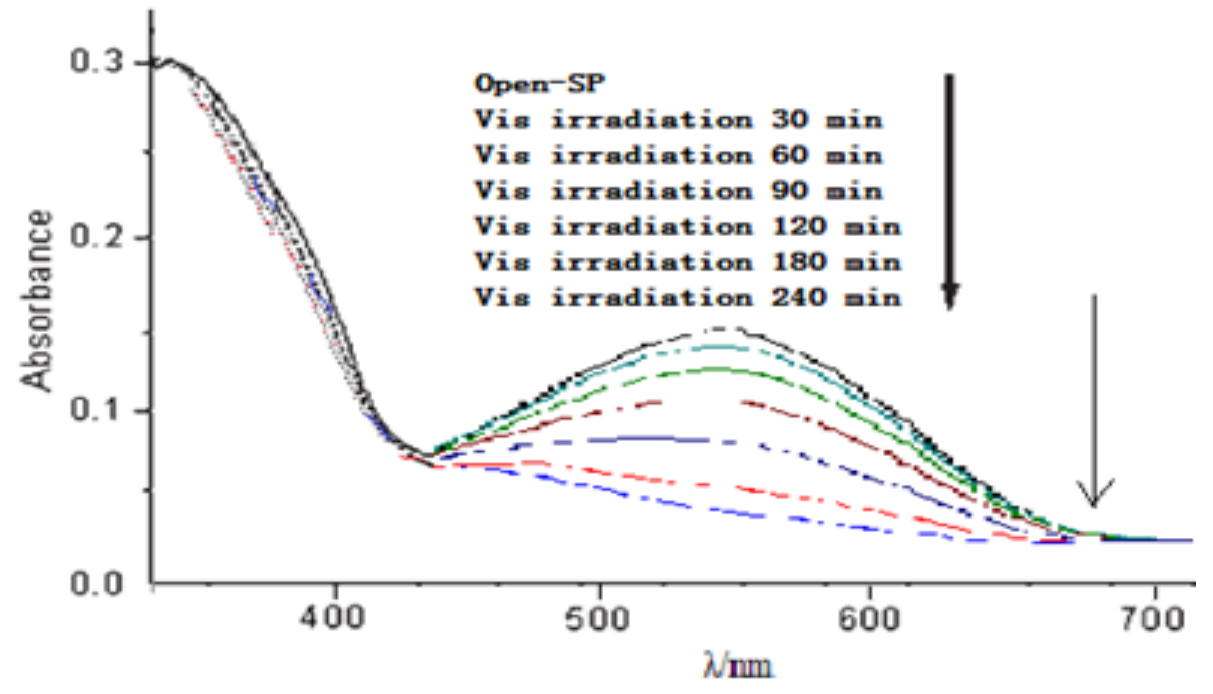

Fig. 4. UV-Vis spectral changes of 3 in ethanol following: left, UV irradiation at $365 \mathrm{~nm}$ for different times, and right, visible irradiation at $620 \mathrm{~nm}$ for different times.

\section{Conclusions}

A novel photoresponsive polymer having spiropyran and chitosan groups was synthesized by simple and easy chemical reaction. The structures of synthesized compounds were confirmed by means of FTIR, H NMR, MS, and SEM. The photochromic properties and the photochemical isomerization were investigated by UV-Vis spectroscopy. The grafted biopolymer showed good photoresponsive properties and had considerable application potentiality in photoresponsive materials. The further application study is underway.

\section{Acknowledgement}

This research was financially supported by the Science and Technology Key Project of Guizhou Province, China (JZ [2015]2006, Z [2015]6013), and Youth Fund Project of Guizhou Academy of Sciences, Guizhou province, China (J [2014]01).

\section{References}

[1]K. Xiao, X. Kong, Z. Zhang, et al., Construction and application of photoresponsive smart nanochannels, J. Photoch. Photobio. C. 26 (2016) 31-47.

[2]G. Petriashvili, M.P. De Santo, L. Devadze, et al., Rewritable optical storage with a spiropyran doped liquid crystal polymer film, Macromol. Rapid. Comm. 37 (2016) 500-505.

[3] B. Ziółkowski, L. Florea, J. Theobald, et al., Porous self-protonating spiropyran-based NIPAAm gels with improved reswelling kinetics, J. Mater. Sci. 51 (2016) 1392-1399.

[4] S. Wan, Z. Ma, C. Chen,et al., A supramolecule-triggered mechanochromic switch of cyclodextrin -jacketed rhodamine and spiropyran derivatives, Adv. Funct. Mater. 26, (2016) 353-364.

[5] K. Kinashi, Y. Miyamae, R. Nakamura, et al., A spiropyran-based X-ray sensitive fiber, Chem. Commun.51 (2015) 11170-11173.

[6] S. Chen, F. Jiang, Z. Cao, et al., Photo, $\mathrm{pH}$, and thermo triple-responsive spiropyran-based copolymer nanoparticles for controlled release, Chem. Commun. 51 (2015) 12633-12636.

[7] R. Klajn, Spiropyran-based dynamic materials, Chem. Soc. Rev. 43 (2014) 148-184. 
[8] L.F. Sukhodub, L.B. Sukhodub, Chitosan for medical application, J. Clin. Exp. Med. Res. 12 (2014) 143-152.

[9] D.S. Duttagupta, V.M. Jadhav, V.J. Kadam, Chitosan: a propitious biopolymer for drug delivery, Curr. Drug Deliv. 12 (2015) 369-381.

[10] M. Kazemzadeh-Narbat, M. Reid, M. S. Brooks, et al., Chitosan nanoparticles as adenosine carriers, J. Microencapsul. 32 (2015) 460-466.

[11] C. Choi, J. Nam, J.W. Nah, Application of chitosan and chitosan derivatives as biomaterials, J. Ind. Eng. Chem. 33 (2016) 1-10.

[12] P. Zou, X. Yang, J. Wang, et al. Advances in characterisation and biological activities of chitosan and chitosan oligosaccharides, Food Chem. 190 (2016) 1174-1181.

[13] H. Tu, Y. Qu, X. Hu, et al., Study of the sigmoidalswelling kinetics of carboxymethylchitosan -g-poly (acrylic acid) hydrogelsintended for colon-specific drug delivery, Carbohyd. Polym. 82 (2010) 440-445.

[14] J.L. Bahr, G. Kodis, L. Garza, et al., Photoswitched singlet energy transfer in a porphyrinspiropyran dyad, J. Am. Chem. Soc. 123 (2001) 7124-7133.

[15] X. Li, Y. Wang, T. Matsuura, and J. Meng, Synthesis of new spiropyrans and spirooxazines having a heteroaromatic pendant and their photochromic behavior, Heterocycles. 51 (1999) 26392651.

[16] W. Norris, Jr. Tyet, S.B. Ralph, Photochromic spiropyrans. I. Absorption spectra and evaluation of the .pi.-electron orthogonality of the constituent halves, J. Am. Chem. Soc. 92 (1970) 1289-1294. 